\title{
AGRICILTIRE
}

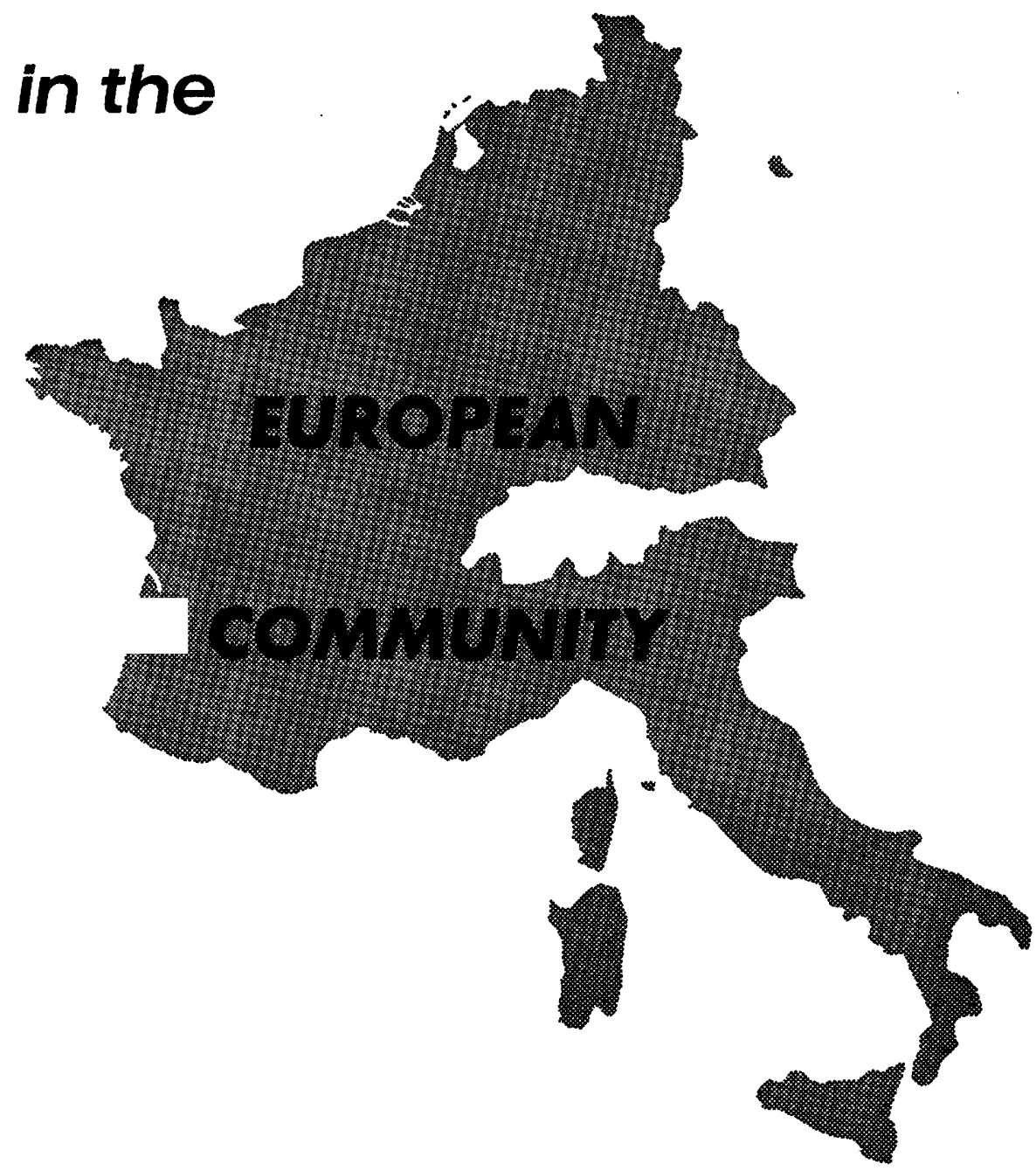

INFORMATION SERVICE OF THE EUROPEAN COMMUNITIES

Division for agricultural Information - Brussels 


\section{AGRICULTURE IN THE EUROPEAN COMMUNITY POPULATION}

TOTAL POPULATION

$k=1,000,000$

France

47,840,000

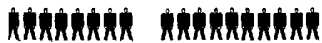

Belgium

$9,290,000$

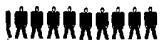

Luxembourg

324,000

Netherlands

$11,967,000$

Wh

Germany (F R)

$57,607,000$

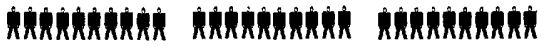

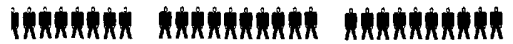

Italy

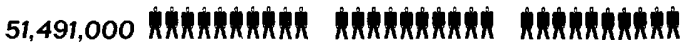

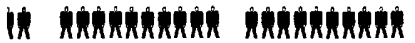

European Community 178,518,000 United States $189,375,000$ United Kingdom $53,673,000$ U.S.S.R.

$224,764,000$

DENSITY (NUMBERS PER sq km) - 1963
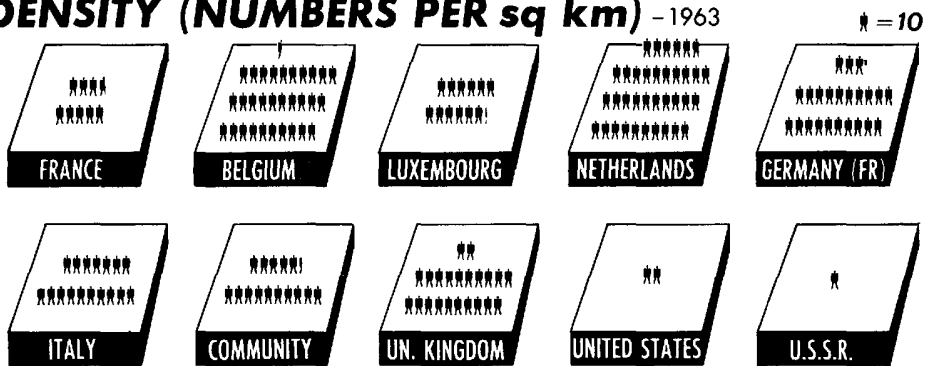

\section{TOTAL WORKING POPULATION - 1962}

In o/o

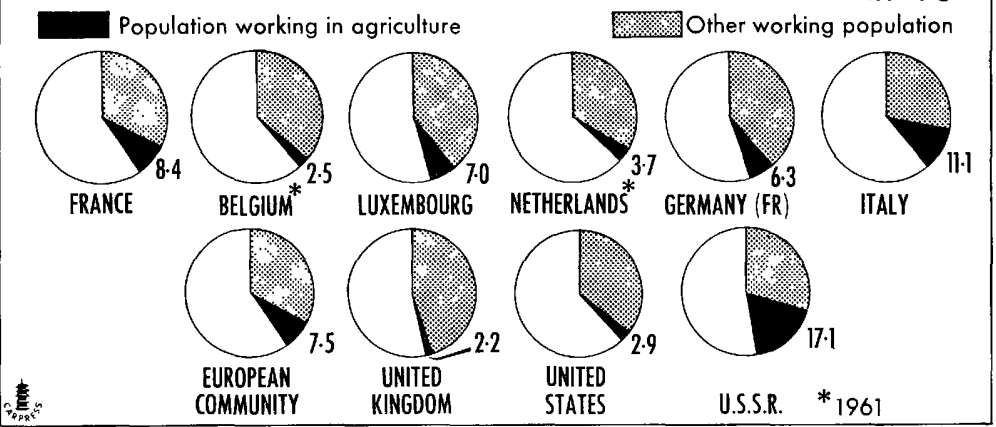




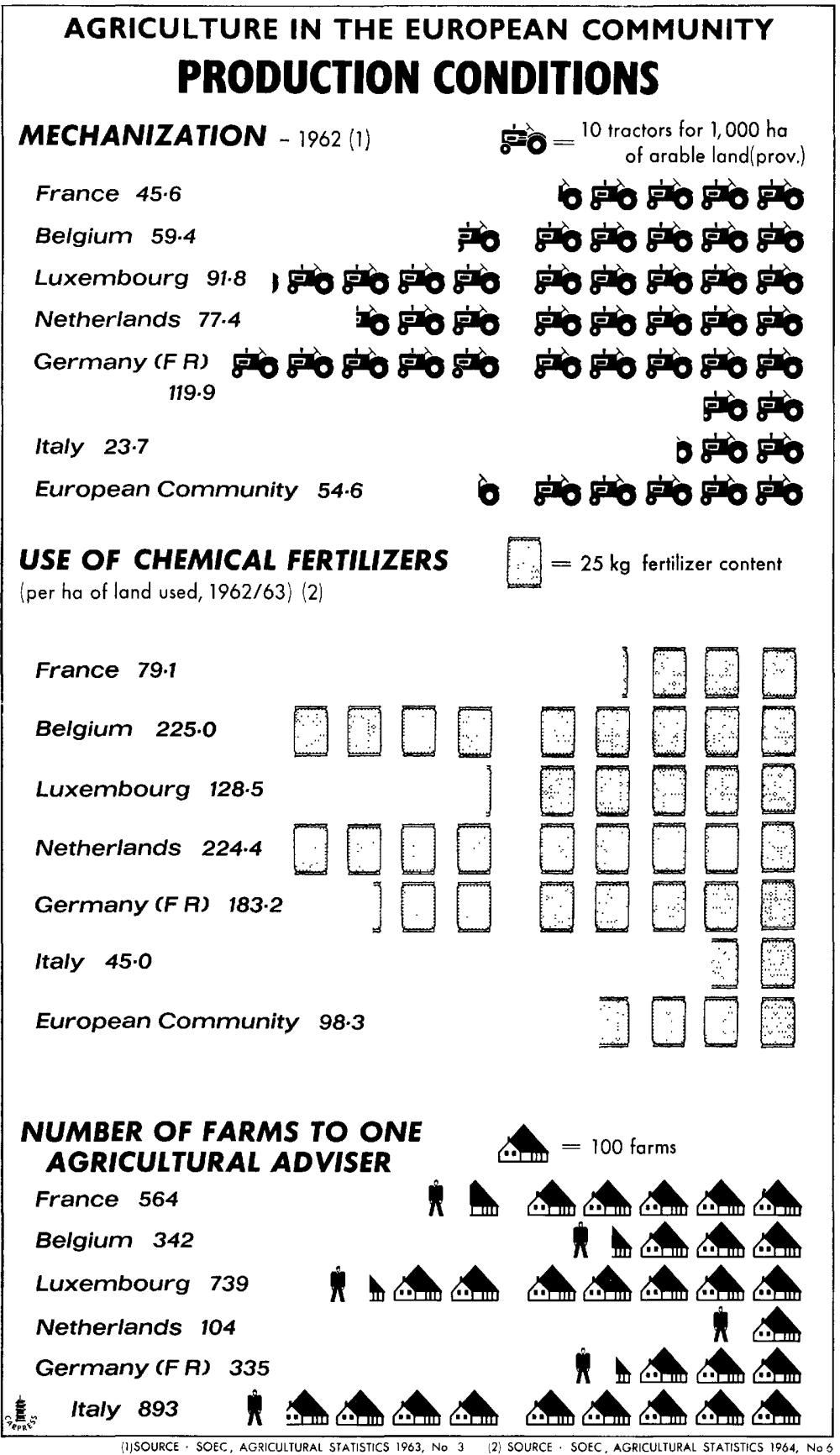




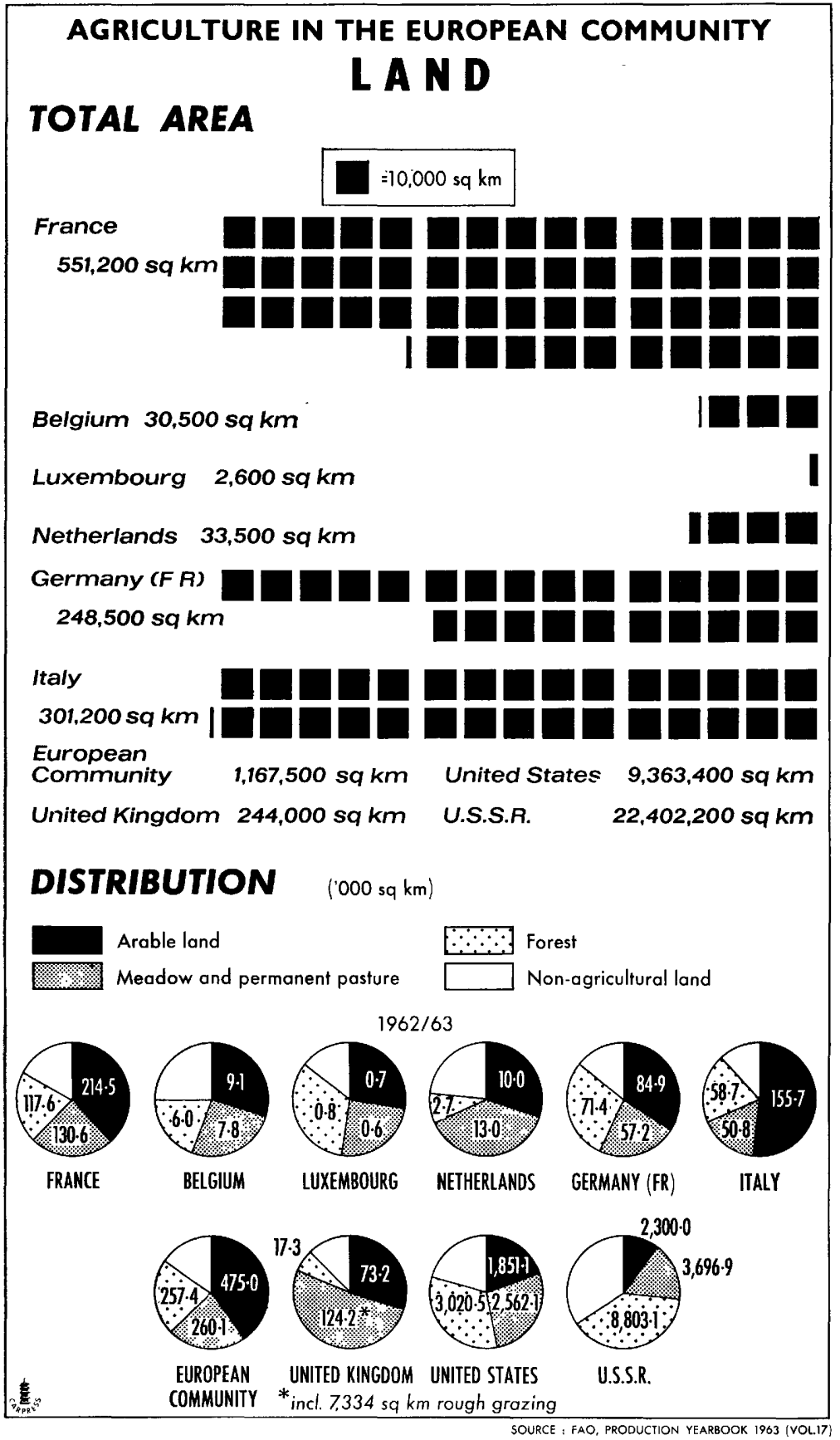




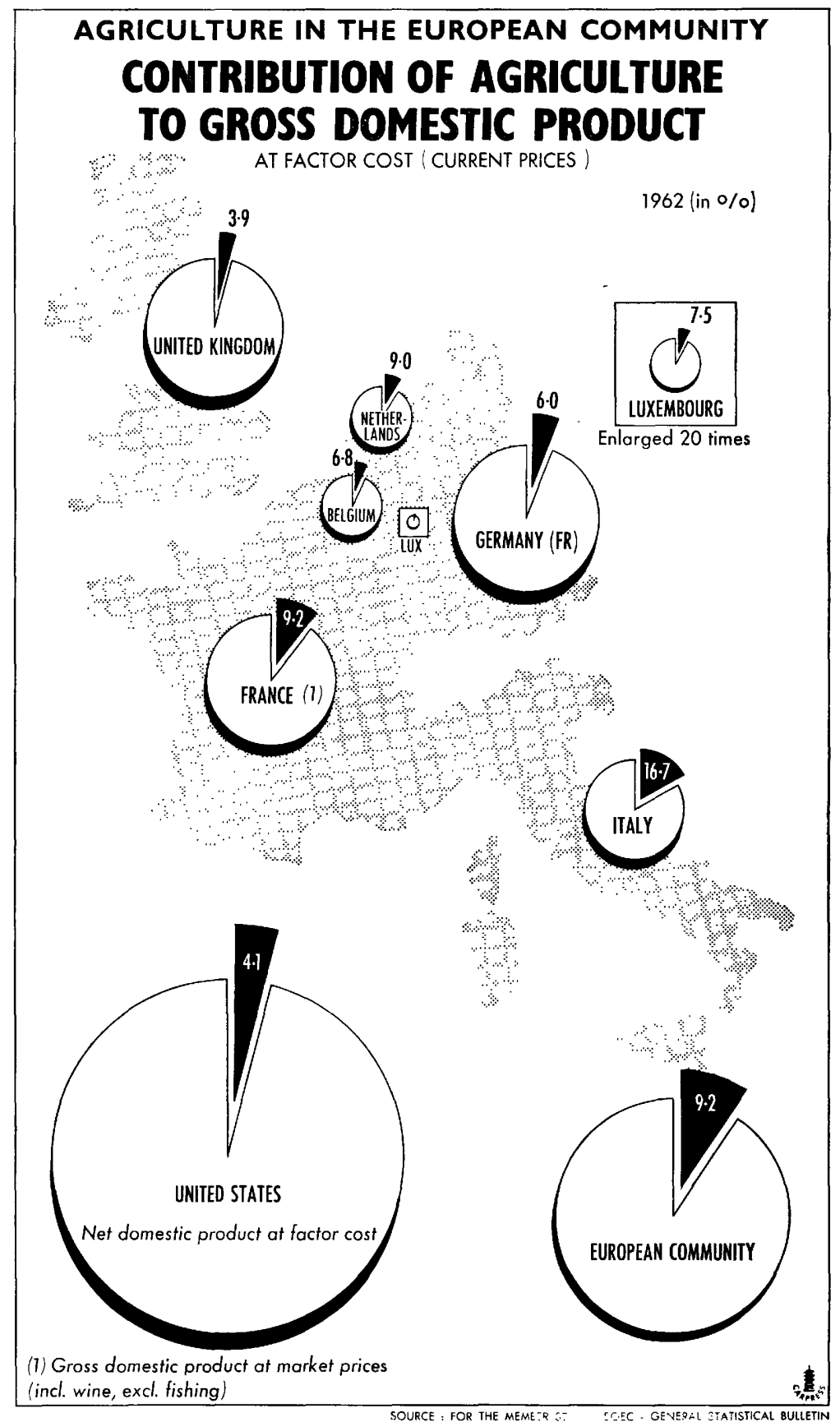




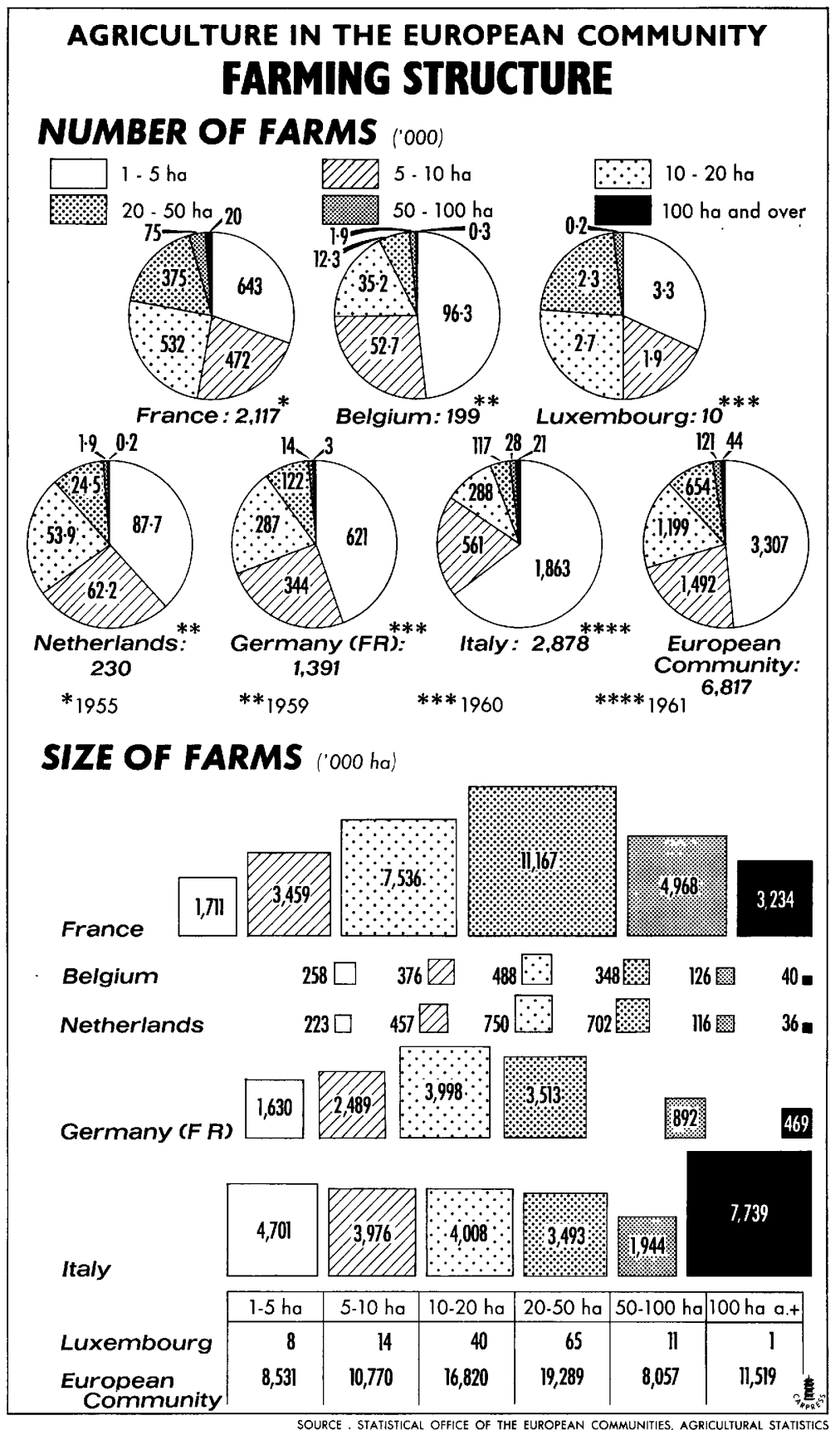




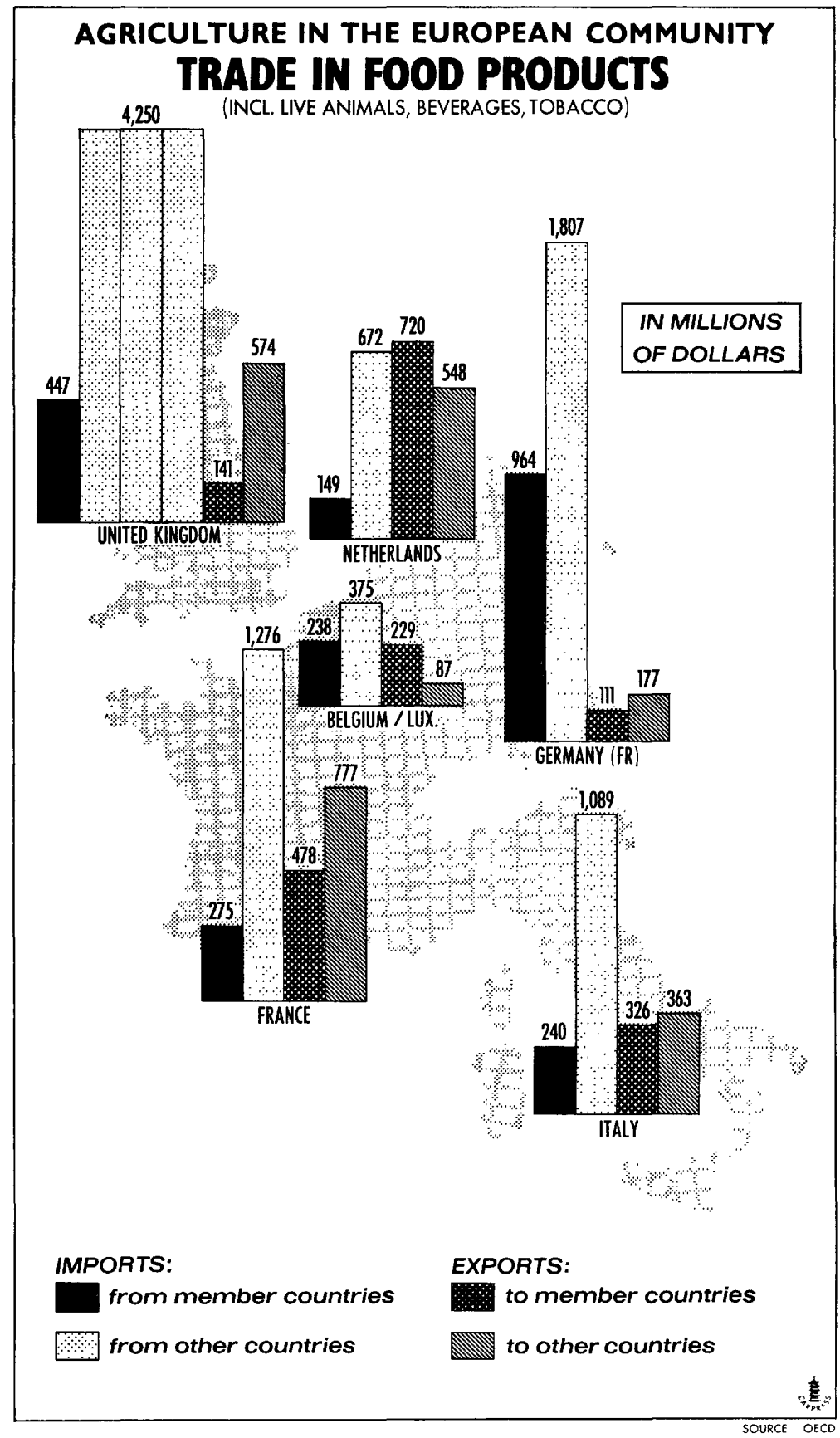




\section{AGRICULTURE IN THE EUROPEAN COMMUNITY BREAKDOWN OF PRIVATE CONSUMERS' EXPENDITURE}

$\square$ Food, Beverages, Tobacco

Rent, Charges, Water, Fuel and light TIIT Furniture, household Goods, Cleaning and Maintenance

France

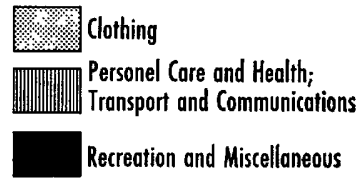

1962

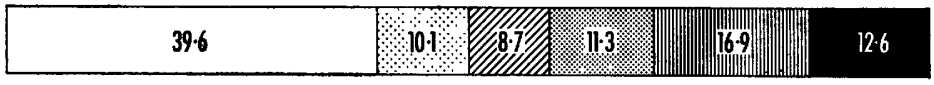

\section{Belgium}

\begin{tabular}{|c|c|c|c|}
\hline 33.9 & 17.0 & Ua & 90 \\
\hline
\end{tabular}

\section{Luxembourg}

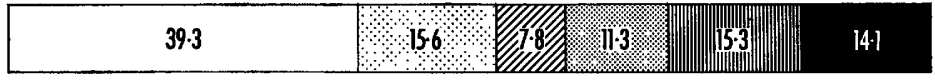

Netherlands

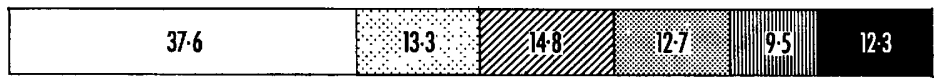

Germany ( $F$ R)

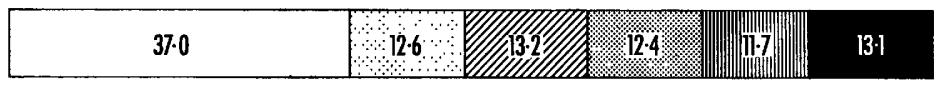

Italy

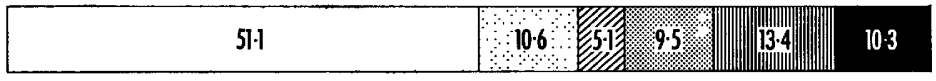

European Community

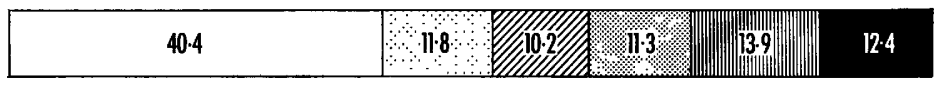

United Kingdom

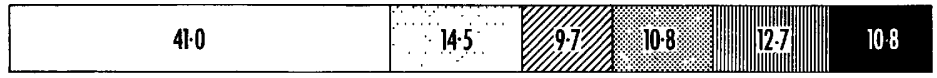

United States

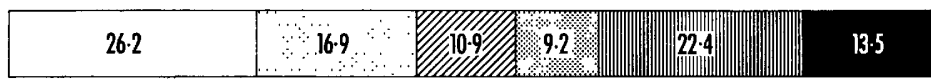

U.S.S.R. 


\section{AGRICULTURE IN THE EUROPEAN COMMUNITY FOOD PRODUCTION AS \% OF NEEDS}

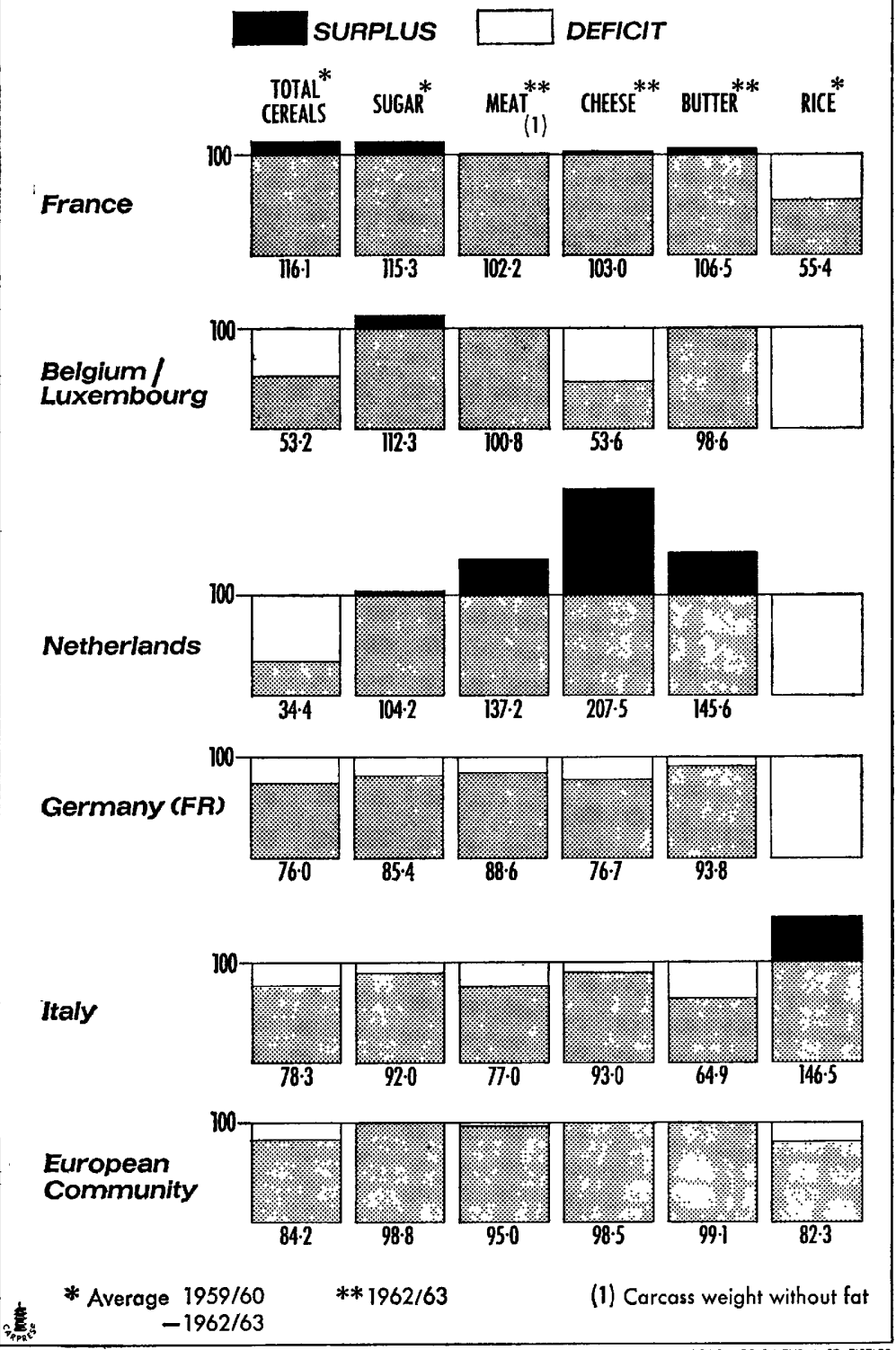




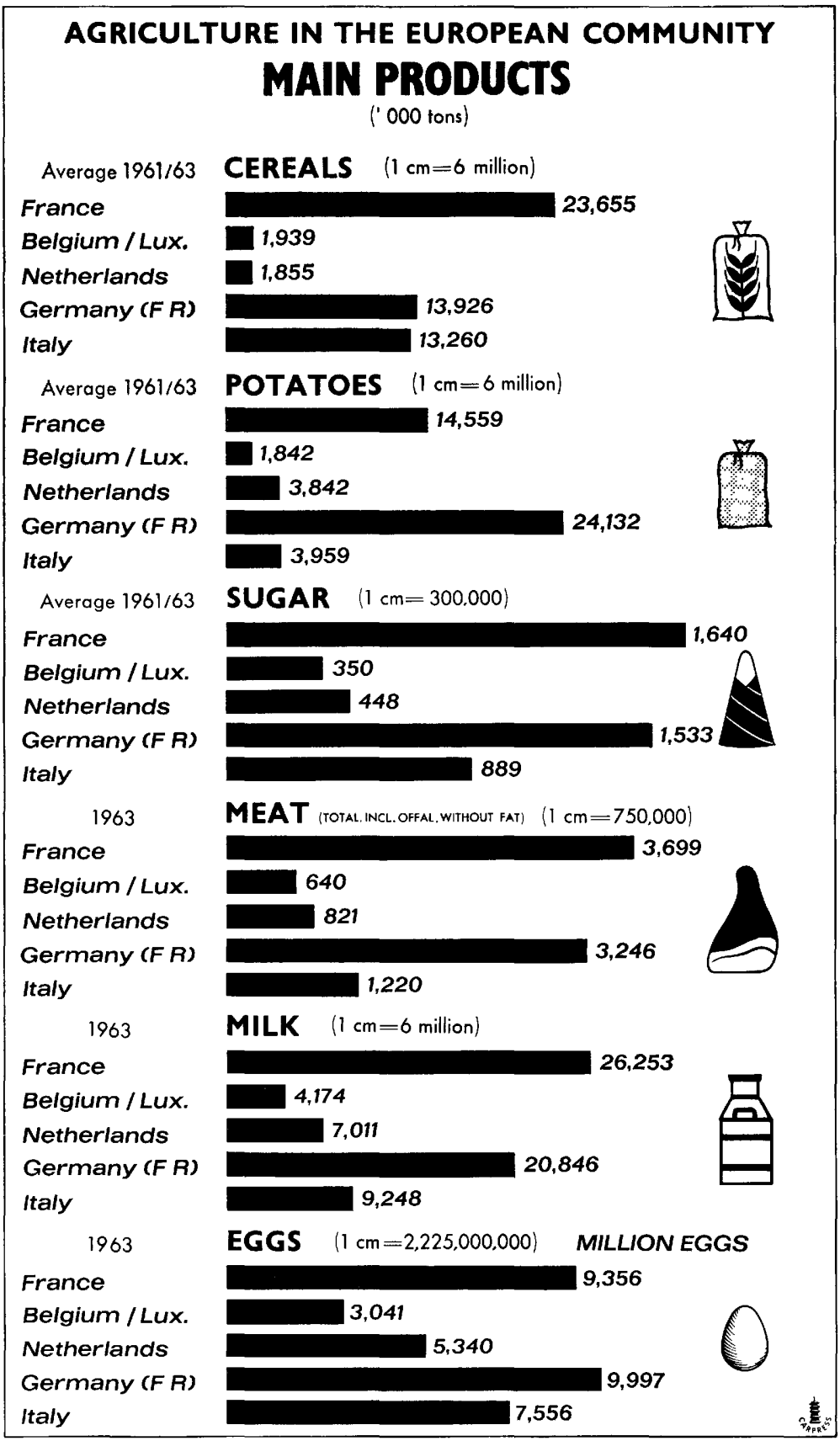




\section{AGRICULTURE IN THE EUROPEAN COMMUNITY WHAT WHEAT IS WORTH}

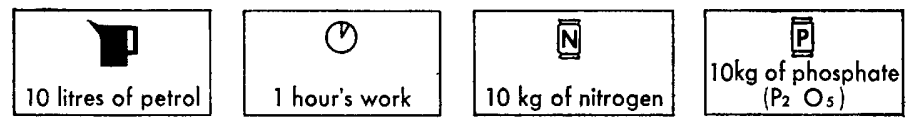

$100 \mathrm{~kg}$ OF WHEAT WILL BUY :

in France

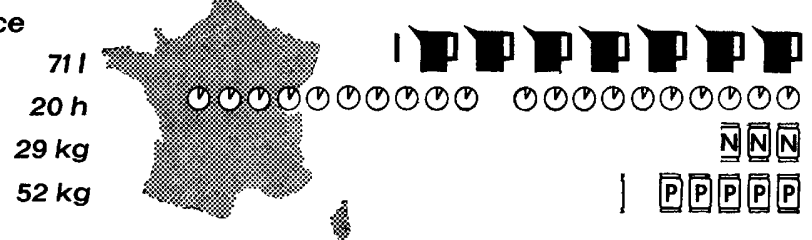

In Belgium

581

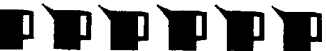

$16 \mathrm{~h}$

1000000000000000

$33.3 \mathrm{~kg}$

$66 \mathrm{~kg}$

佥馬

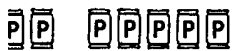

in Luxembourg ${ }^{*}$

$38 \mathrm{~kg}$

$125 \mathrm{~kg}$

In the Netherlands

661

$12 \mathrm{~h}$

$32 \mathrm{~kg}$

$47 \mathrm{~kg}$

in Germany (FR)

711

$17 \mathrm{~h}$

$40 \mathrm{~kg}$

$66 \mathrm{~kg}$

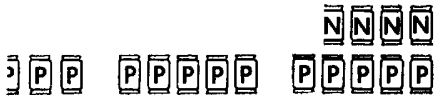

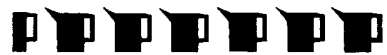

OO 0000000000

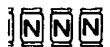

量回回回回

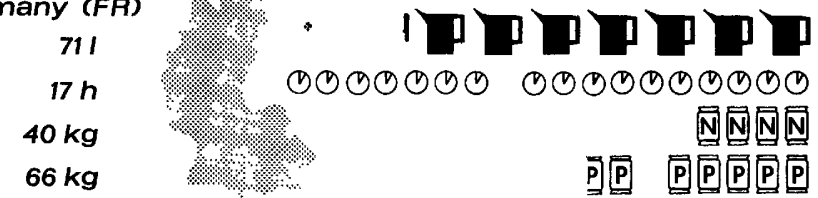

in Italy

711

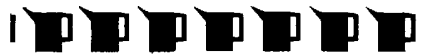

$22 h 0000000000000000000000$ $41 \mathrm{~kg}$

$71 \mathrm{~kg}$

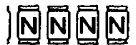

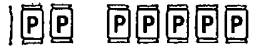




\section{INFORMATION SERVICE \\ OF THE EUROPEAN COMMUNITIES}

\section{BRUXELLES :}

244, rue de la Loi

\section{LUXEMBOURG :}

35.00 .40

$$
18 \text {, rue Aldringer }
$$

292.41

BONN :

$$
\text { Zitelmannstrasse, } 11
$$

DEN HAAG :

Alexander Gogelweg, 22

PARIS :

61, rue des Belles-Feuilles, $16^{\text {tme }}$

KLEber53-26

ROMA :

via Poli, 29

LONDON :

$670.696 / 688.182$

23, Chesham Street, S.W. 1.

WASHINGTON :

Belgravia 4904

Farragut Building 808 900-17th Street

296-5131

NEW YORK :

Commerce Building 2207

155 East 44th Street

GENEVE :

212 MU 20458

72 , rue de Lausanne 